\title{
Industrielle Cybersicherheit: ein Wachstumsmarkt
}

Fluggesellschaften, Telekommunikationsdienstleister, Handelshäuser, Industrieunternehmen - kein Wirtschaftszweig ist von der Bedrohung durch Hackerangriffe ausgenommen. Die öffentliche Aufmerksamkeit ist dann besonders hoch, wenn Kundendaten betroffen sind oder wenn durch Ransomware-Systeme komplett lahmgelegt werden. Dabei sind solche Cyberattacken für das betroffene Unternehmen meist „nur“ Image-schädigend und kostentreibend; selten sind sie mit existenziellen Risiken verbunden.

Anders verhält es sich bei Cyberangriffen, die auf Produkte oder den Betrieb von Unternehmen abzielen. Hier werden Produkt- und Betriebssicherheit sowie unter Umständen auch ganze Geschäftsmodelle gefährdet. Die Umsetzung von IIoT-Strategien und die daraus resultierende voranschreitende Vernetzung von Industrieanlagen und Infrastruktursystemen verstärken dabei die Herausforderungen bezüglich der Cybersicherheit noch mehr. Vielen Unternehmen fehlen jedoch die Kompetenzen und die Ressourcen, um ausreichend interne Sicherheitsmaßnahmen zu ergreifen.

Dementsprechend wächst der Markt für industrielle Cybersicherheit. Im Gegensatz zum herkömmlichen Fokus auf Datenschutz und -sicherheit zielt dieses Marktsegment auf den Schutz von Produktions- und Lieferketten vor unbefugtem Zugriff, Manipulation oder gar Unterbrechung. Neben Lösungen zur Abwehr solcher Risiken werden zunehmend Security-by-DesignAnsätze verfolgt, um schon beim Aufbau von Infrastrukturen die Risiken zu minimieren. Dabei wird offensichtlich, dass funktionsorientierte Cybersicherheitsbemühungen in Unternehmen und Organisationen mittlerweile keinen nachhaltigen Ansatz für Cybersicherheit mehr darstellen. Unternehmen müssen neue integrierte Strategien entwickeln, die IT-, OT- und IIoT-Sicherheitsbemühungen kombinieren und die Nutzung aller Cybersicherheitsressourcen des Unternehmens maximieren. Der Markt für industrielle Cybersicherheit wird also weiterhin wachsen.

Ihre ZWF-Herausgeber und-Redaktion

Become the Engineer of Tomorrow

Die Deutsche Akademie für Digitale Bildung (DADB) ist Anbieter eines innovativen Blended Learning Systems mit hochwertigen, technologieorientierten Lerninhalten, die mit Hilfe von Augmented und Virtual Reality illustrativ ansprechend und unterhaltsam vermittelt werden.

Mit Hilfe unser renommierten Contentpartner aus Wissenschaft und Industrie transportieren wir „German Engineer- ing“ global an Hochschulen in aufstrebenden Wachstumsmärkten. Damit bieten wir dort einer jungen Generation die Möglichkeit, ihre Zukunft aktiv mitzugestalten und den Anforderungen an den Ingenieur von morgen gerecht zu werden. Für weitere Informationen zur DADB und Möglichkeiten einer Zusammenarbeit als Contentparter: www.da-db.com info@da-db.com

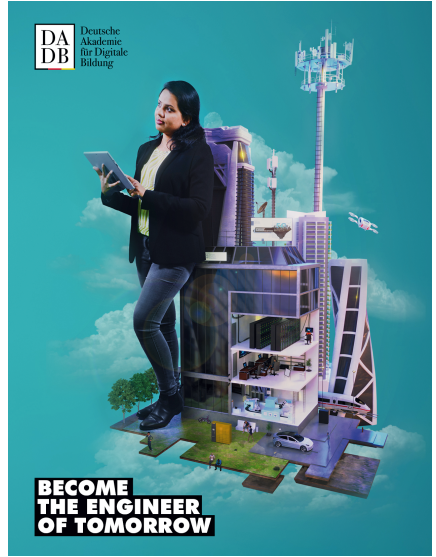

